\title{
Interplay of network structure and dynamics in functional organization of the visual cortex
}

\author{
Yudy Carolina Daza C.* \\ Statistical and Interdisciplinary Physics Group, National Scientific and Technical Research Council (CONICET), \\ Centro Atómico Bariloche 8400, Argentina \\ Pablo M. Gleiser \\ Medical Physics Department, National Scientific and Technical Research Council (CONICET), Centro Atómico Bariloche 8400, Argentina \\ Francisco A. Tamarit \\ Theory of Condensed Matter Group, National Scientific and Technical Research Council (CONICET), FaMAF, \\ Universidad Nacional de Córdoba 5000, Argentina
}

(Received 8 June 2018; revised manuscript received 12 October 2018; published 10 December 2018)

\begin{abstract}
The functional patterns of the visual cortex observed in some mammals such as cats, primates, and humans has allowed us to understand basic principles of organization in the structure of the cortex. However, the observation of different kinds of functional arrangement in other animals such as mice, rats, rabbits, and squirrels, called salt and pepper patterns, raise questions about which of these principles can be transferred to the understanding of visual processing in general. In order to gain insight into these basic principles, in this work we propose a simple model for the formation of spatiotemporal patterns in the visual cortex. The model is based on coupled phase oscillators that interact through an evolving complex network that is embedded in a two-dimensional Euclidean space. In this way we are able to explore the relation between network structure and functional organization. We find that the model allows for the emergence of clustered synchronized states that are spatially segregated as some orientation maps, and also synchronized states that are spatially interspersed, resembling salt and pepper organizational maps.
\end{abstract}

DOI: 10.1103/PhysRevE.98.062307

\section{INTRODUCTION}

Many functional phenomena in the cortex can be understood in terms of the global activity of a large number of elements that interact in a nonlinear way. Examples include, among others, activity maps, which are spatially ordered representations of functional response properties [1], orientation preference maps (OPM), that arise from groups of neurons that synchronize their activity in presence of stimuli of an specific orientation, and also ocular dominance maps corresponding to groups of neurons that synchronize their activity in presence of stimuli coming from the same eye.

Recently, we proposed a simple model [2,3] that can retrieve patterns of activity (OPM) observed in the visual cortex in different mammals species [4-9]. The model is based on coupled phase oscillators that interact through a complex network that is embedded in a two-dimensional Euclidean space by the method proposed by Rozenfeld et al. [10,11]. This allows us to consider physical distances between nodes and then, the implementation of a geographical distancedependent form of interaction [12-16].

In this work, we extend the ideas presented in Refs. [2,3] in order to explore further the relation between network structure and functional organization. In particular, we incorporate an important biological characteristic of the cortex networks: their plasticity [17]. The term plasticity refers to changes

\footnotetext{
*yudy.daza@cab.cnea.gov.ar
}

in the connections due to synaptic modifications mediated by changes in the number of neuroreceptors, the number of neurotransmitters or, in the way how the synapses responds. This characteristic can be implemented in the model including synaptic modifications for the connections in the network. If the synaptic modification is formulated in a way that evolves jointly with the dynamics, the structural properties of the network can be related with their dynamical properties [18-21]. This can be achieved by means of rules that introduce adaptation in the synapses of the network. This rules have to reflect the way in which the synaptic efficiency between two neurons is affected by the concomitance of their activity [22].

As expected, we find that functional clusters can be related to spatially located groups of synchronized oscillators when local networks are considered. Surprisingly, we also found that functional clusters can be found when random networks with no spatial organization are taken into account. This situation resembles a particular kind of functional organization observed in mammals in some species of mice and squirrels, the so-called salt and pepper patterns [23-25], where the maps of neural activity show little order and the orientation selectivity appears interspersed [26].

\section{MODEL}

In a cortical tissue, the morphology, physiology and the circuits between neurons vary with the position in the cortex. This arrangement of the circuitry between neurons and the 
relation of the interaction with the spatial localization of the neurons, makes necessary to consider a way to take into account the physical distance between neurons. This can be accomplished by embedding a complex network in a twodimensional Euclidean space. This allows for the introduction of an interaction function that varies with distance. Recently, we proposed a simple model that includes this characteristic, and allows for the emergence of patterns like the ones observed in the visual cortex [2,3]. In particular, we analyzed the behavior of a Kuramoto model of coupled phase oscillators [27-29] with competing interactions in a complex network embedded in two-dimensional Euclidean space [3].

Our results show that even with this simple approximation the model is still able to capture the main ingredients of the cortical organization. In fact, with only a few parameters, the model allows for the emergence of clustered or striped patterns that quantitatively resemble experimental patterns observed in infant macaques, monkeys, and ferrets [3].

We extend this model by introducing the time changing nature of the neural circuits in the cortex. Now the neurons interact in a way that enable that the weights in the network connections vary and in this way can module the form in which the stimuli are perceived [30]. In order to introduce this characteristic, we focus on the ideas presented by Assenza et al. [31] where a general adaptive network can be obtained by means of the interplay of two hypothesis: the plasticity of the network, and a mechanism that seeks to establish an equilibrium in the system. The plasticity can be interpreted as a learning process where the network reinforces the connections that have similar dynamic states. However, this mechanism has to be balanced by a conservation of the total wiring, and thus depends on the total availability of resources. In the present case, the degree of local synchronization achieved during a period of time $T$, called the memory of the system, is measured. In each moment the synchronization $p_{i j}^{T}(t)$ of a couple of oscillators $i$, and $j$, is compared with the mean synchronization of the population of the neighbors of node $i$. If this synchronization is higher than the average of the population, the actual connection enhances its strength. On the contrary, if the synchronization is lower, then the connection is not strong enough to overcome the influence of the others neighbors and weakens.

The changing weights can be included in a Kuramoto model for coupled oscillators in order to obtain a changingin-time system as in Eq. (1):

$$
\dot{\theta}_{i}(t)=\left[\omega_{i}-\sum_{j=1}^{V_{i}} F_{i j}\left(\theta_{i}, \theta_{j}\right)\right] .
$$

Where $F_{i j}\left(\theta_{i}, \theta_{j}\right)$ is an interaction kernel given by:

$$
F_{i j}\left(\theta_{i}, \theta_{j}\right)=\frac{\lambda}{V_{i}} W_{i j}(t) \sin \left[\theta_{j}(t)-\theta_{i}(t)\right] .
$$

$W_{i j}$ is the weight of the connections between oscillators $i$ and $j$, and the sum is limited to the neighborhood ( $V_{i}$ oscillators) of the $i$ th node. The variable $\lambda$ is the global coupling parameter. In the absence of adaptation this coupling parameter rules alone the synchronization between the oscillators.

The kernel in Eq. (2) can be useful for adding a new ingredient to the interaction, such as the dependence with the physical distance. As a first step in this direction we considered a simple nonlinear term, which allows the strength of the interaction to decrease with distance. In particular we choose a functional form that decays as a power law, with the inverse of the Euclidean distance between two nodes. This can be achieved by modulating a single parameter $\gamma$. In this case, the functional form of the kernel in Eq. (2) takes the form:

$$
F_{i j}\left(\theta_{i}, \theta_{j}\right)=\frac{\lambda}{V_{i}} \frac{1}{d_{i j}^{\gamma}} W_{i j}(t) \sin \left[\theta_{j}(t)-\theta_{i}(t)\right],
$$

where $d_{i j}$ is the Euclidean distance:

$$
d_{i j}=\sqrt{\left(x_{j}-x_{i}\right)^{2}+\left(y_{j}-y_{i}\right)^{2}} .
$$

In the following section we will analyze this model by considering three different underlying structure of connections, given by a local, a random, and a scale-free network. Our goal is to characterize the dynamical state of the system and to establish a relation with the underlying network structure.

\section{RESULTS}

The Kuramoto formula Eq. (1) together with the plasticity of the weights the constitute a system of equations for the coevolution of the states of the nodes and the network, given initial conditions of phase, frequencies, synaptic strengths and connectivities. We used fourth-order Runge-Kutta technique to integrate the equations, given a uniform initial distribution of phases $(\theta)$ in the $[0, \pi]$ interval and also a uniform distribution for the natural frequencies $(\omega)$ in the $[-0.5,0.5]$ interval.

\section{A. Local topology}

The first connectivity scheme to be considered for the oscillators is a local network, where the nodes have a mean number of 12 neighbors. In order to study the global synchronization of the system we consider the Kuramoto order parameter [27,29], given by:

$$
\mathbf{r}(t) e^{i \Theta(t)}=\frac{1}{N} \sum_{j=1}^{N} e^{i \phi_{j}(t)} .
$$

In order to examine quantitatively how the order of the system is modified by the adaptation dynamics we allow the system to evolve until the order parameter $r$ reaches a stationary state. In Fig. 1 we plot the order parameter $r$ as a function of time $t$, for different values of the function of memory $T$ and the global coupling $\lambda$. All cases correspond to $\gamma=1.5$, and a maximum of $t=25000$ integration steps.

Three different regimes can be observed: an incoherent state, with a low mean value of the order parameter (bottom row); an intermediate regime with partial synchronization, characterized by large fluctuations of the order parameter (middle row); and an ordered regime characterized by a large value of the order parameter that oscillates around a well-defined mean value (top row). When the value of $\gamma$ is increased (decreased) the order parameter reaches the ordered regime for higher (lower) values of $\lambda$.

In order to explore the relation between the different dynamical states of the system and the underlying network structure, we analyze first the community structure, then the 


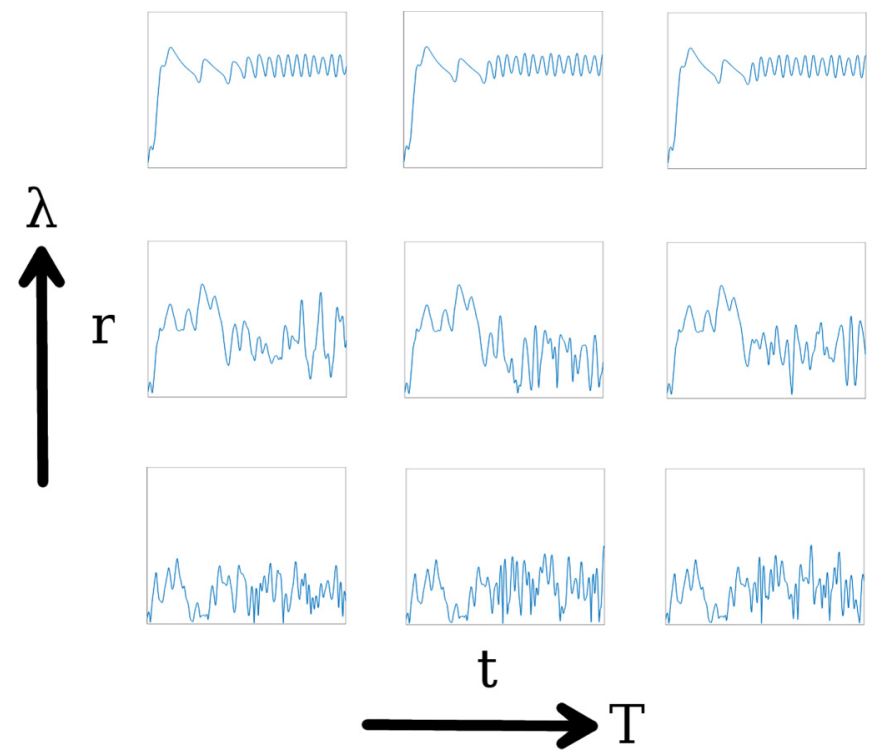

FIG. 1. Order parameter $r$ as a function of time $t$ for an initial local network, varying the memory $T$ (from left to right $T=100,200,300$ ) and the coupling $\lambda$ (from bottom to top $\lambda=$ $1,1.2,1.4)$

local synchronization, and finally establish a relation between the synchronization regimes and the spatial localization of the nodes.

The network communities were determined using the Louvain method [32]. In Fig. 2 we use different colors to identify the communities, and plot the nodes in their spatial location due to the embedding. All the figures correspond to simulations using the same initial configuration. In the bottom
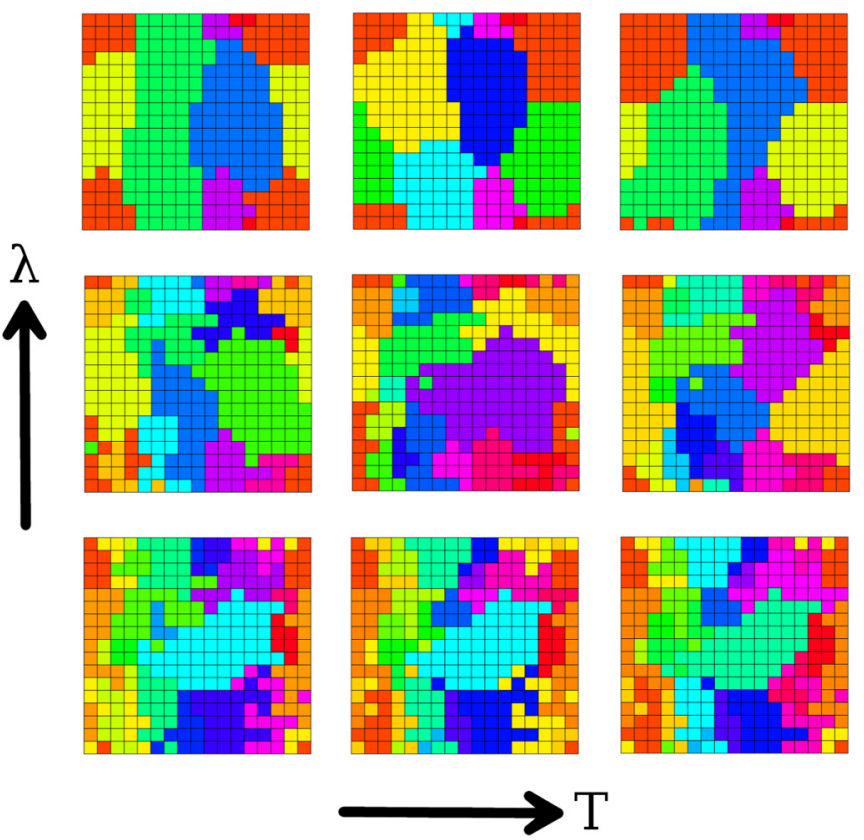

FIG. 2. Communities of interconnected nodes for an initial local network of connections varying the memory $T$ (from left to right $T=100,200,300$ ) and the coupling $\lambda$ (from bottom to top $\lambda=1,1.2,1.4$ ).

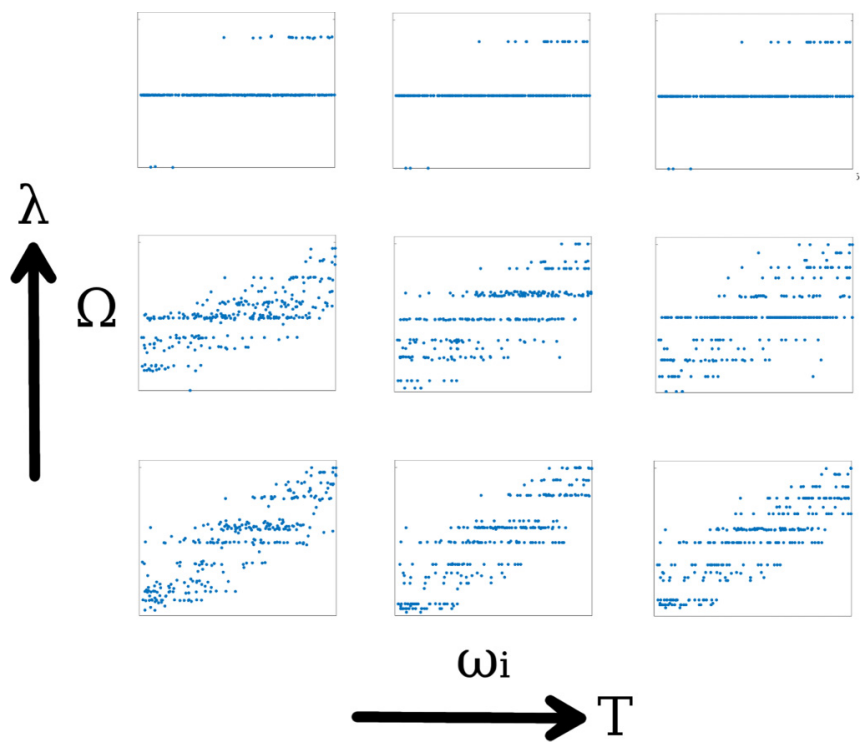

FIG. 3. Average frequency $\Omega$ as a function of the initial frequency $\omega$ for an initial local network of connections, varying the memory $T$ (from left to right $T=100,200,300$ ) and the coupling $\lambda$ (from bottom to top $\lambda=1.0,1.2,1.4$ ).

row we find that the system is divided in a number of small communities. As $\lambda$ is increased the size of some communities grow, and thus the total number of communities decreases. When the value of $\gamma$ is increased (decreased), and thus the interaction decays faster (slower) with distance, a similar qualitative behavior is observed. However, in order to obtain the same number of communities it is necessary to increase the value of the coupling $\lambda$.

The order parameter gives information on the global synchronization of the system. Since the system is clearly divided in clusters, we will also analyze the local synchronization of the system. In order to do this we plot in Fig. 3 the average frequency $\Omega$ as a function of the initial frequency $\omega$. For all the cases considered above we find the presence of synchronized clusters. They appear as horizontal lines, which reveal the presence of groups of oscillators that have changed their natural frequency to the same average frequency. When $\lambda=$ 1.0 a large number of synchronized clusters can be observed. The larger clusters are centered around zero, and decrease their size as $\Omega$ approaches 1 and -1 . As $\lambda$ is increased the number of clusters is reduced and their size becomes larger. Eventually, for sufficiently large values of $\lambda$ a single giant cluster centered around zero emerges.

In order to establish a direct relation between the local synchronization (Fig. 3) and the network structure (Fig. 2), we plot in Fig. 4 the nodes in their corresponding spatial location, and use colors to denote their average frequency $\Omega$. By comparing Fig. 2 and Fig. 4 it can be seen that when $\lambda=1.0$ (bottom row) there is an almost direct relation between the community structure and the corresponding average frequency. The spatial localization plays a prominent role in the synchronization regime, and each community has a different $\Omega$. As $\lambda$ is increased the number of synchronized clusters decreases, and different communities share the 


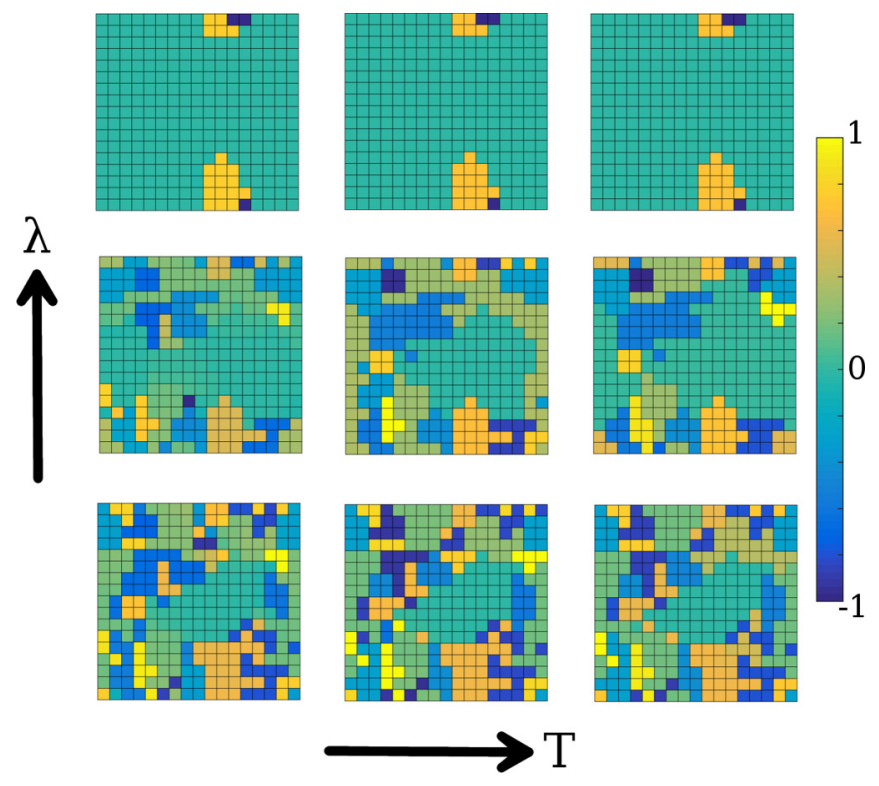

FIG. 4. Spatial location of nodes with average frequency $\Omega$ for an initial local network of connections, varying the memory $T$ (from left to right $T=100,200,300$ ) and the coupling $\lambda$ (from bottom to top $\lambda=1,1.2,1.4$ ).

same $\Omega$ (middle row). Eventually, for sufficiently large $\lambda$ all the communities share the same $\Omega$ (top row).

Finally, we examine the phases of the oscillators, and see how their synchronization is influenced by the underlying network structure. In Fig. 5 we plot a snapshot of the phases in their corresponding spatial localization. In all cases, the presence of spatially located clusters that share the same phase can be observed. When $\lambda=1.0$ (bottom row) the system is in an incoherent state, as was already seen in the time

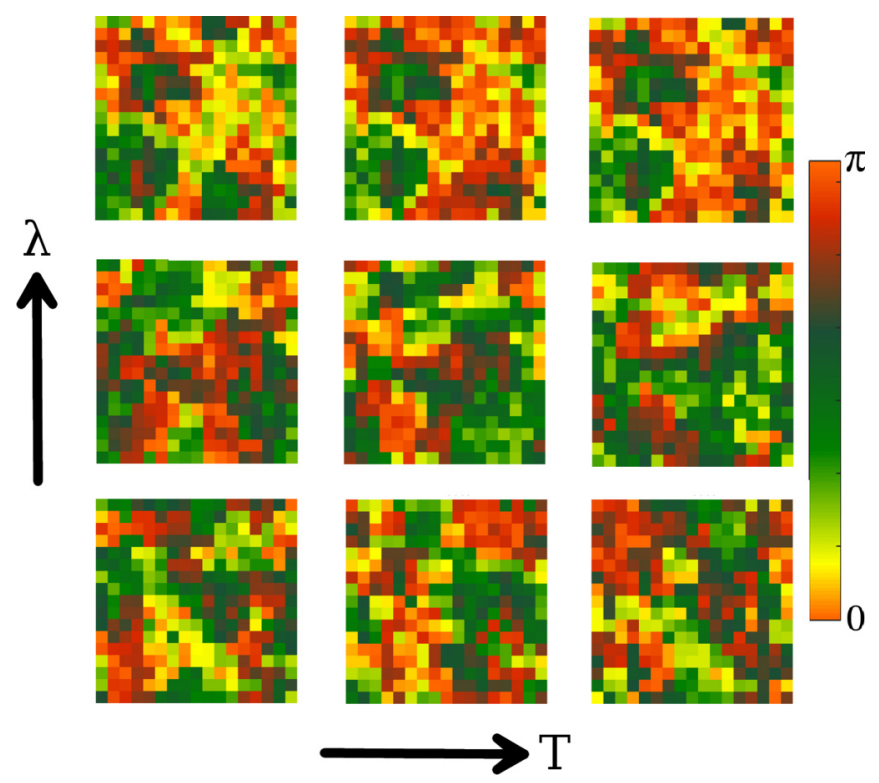

FIG. 5. Snapshot of the phases for an initial local network of connections varying the memory $T$ (from left to right $T=$ $100,200,300$ ) and the coupling $\lambda$ (from bottom to top $\lambda=$ $1,1.2,1.4)$.

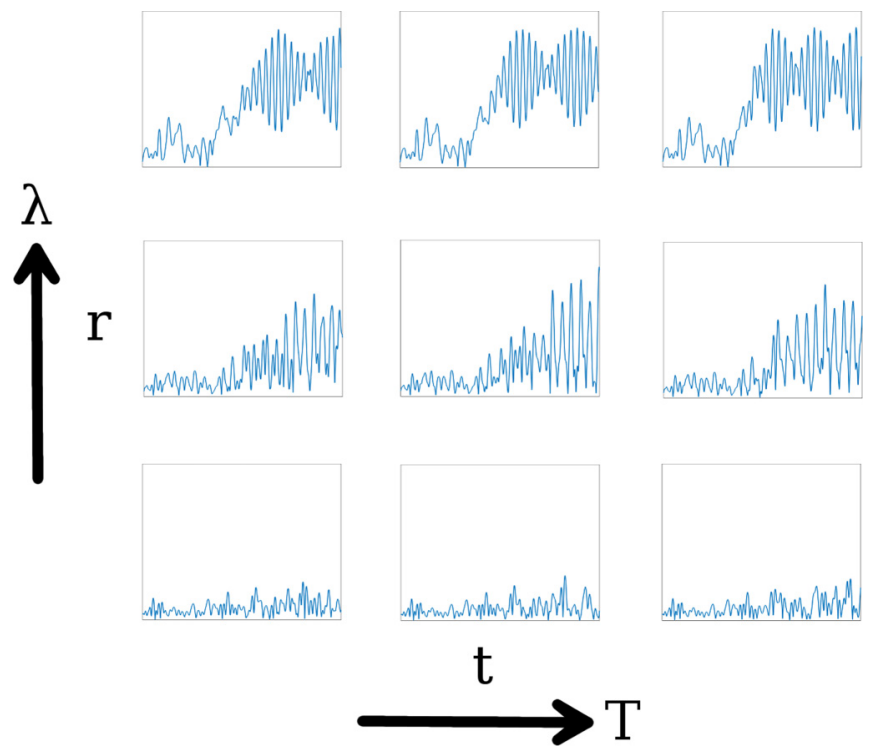

FIG. 6. Order parameter $r$ as a function of time $t$ for an initial random distribution of connections varying the memory $T$ (from left to right $T=150,180,250$ ) and the coupling $\lambda$ (from bottom to top $\lambda=1.0,3.0,5.0)$.

evolution of the global order parameter $r$ (Fig. 1). We can see now that this is due to the presence of many small clusters, that are locally synchronized, and whose competition clearly hinders global synchronization. As $\lambda$ is raised, these clusters synchronize, leading to an increase in global synchronization (Fig. 1). Note however, that the phase differences between clusters can be large, thus leading to large fluctuations in $r$. Eventually, as $\lambda$ is raised further, the differences between the clusters diminish, leading to a growth, and also to a reduction of the fluctuations in the global order parameter $r$.

\section{B. Random topology}

Now, we consider a different connectivity scheme, where the neighborhood of each node is randomly selected over all the network. As in the previous section we let the system evolve until the order parameter $r$ reaches a stationary state. In Fig. 6 we plot the time evolution of the global order parameter $r$ as as a function of memory $T$ and global coupling $\lambda$. All cases correspond to $\gamma=1.5$, and a maximum of $t=25000$ integration steps.

Again, we observe three different regimes: an incoherent state, with a low mean value of the order parameter (bottom row); an intermediate regime with partial synchronization, characterized by large fluctuations of the order parameter (middle row); and an ordered regime characterized by a large value of the order parameter with an oscillating periodic behavior (top row). When the value of $\gamma$ is increased (decreased), and thus the interaction decays faster (slower) with distance, a similar qualitative behavior is observed. However, in order to obtain the same behavior it is necessary to increase the value of the coupling $\lambda$.

Next, we analyze the community structure using the Louvain method [32]. In Fig. 7 we plot the nodes in their corresponding spatial location due to the embedding. All the figures 

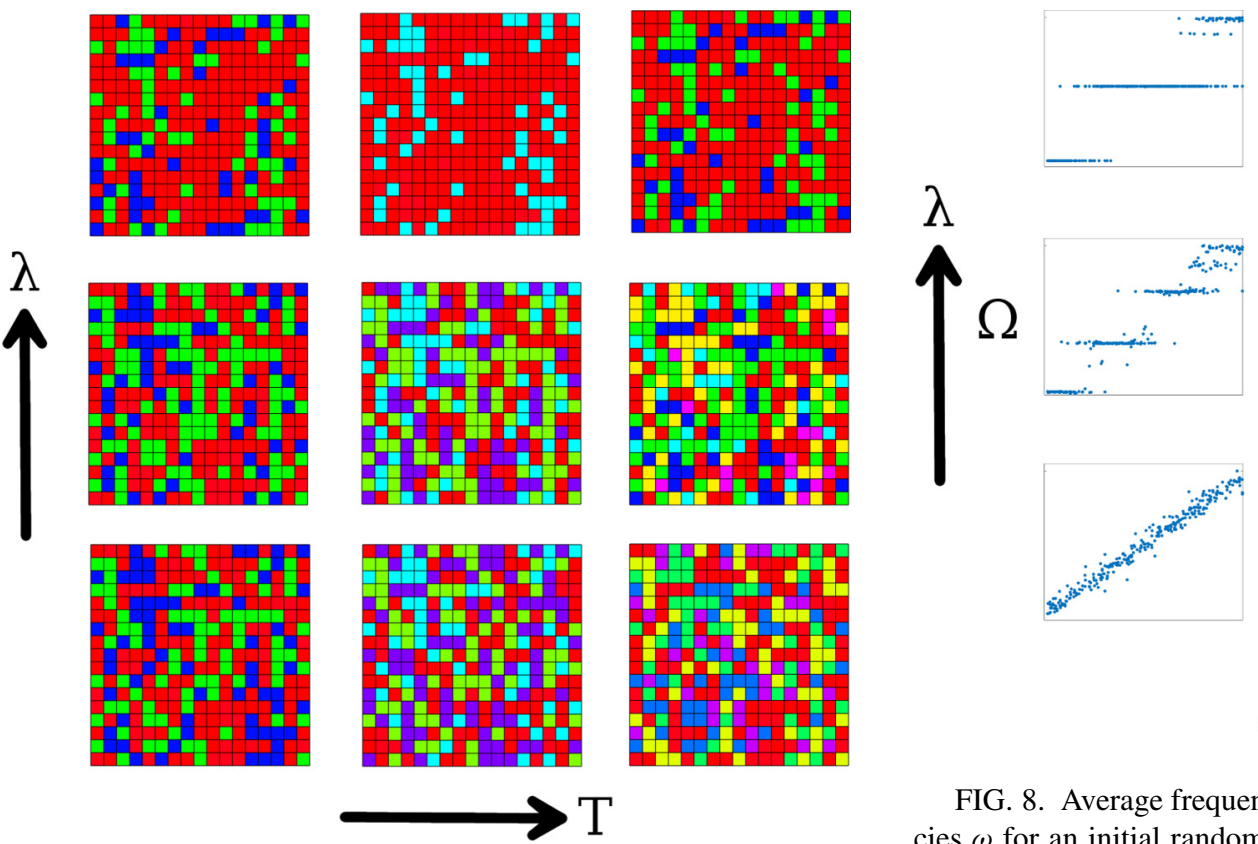

FIG. 7. Communities of interconnected nodes for an initial random distribution of connections varying the memory $T$ (from left to right $T=150,180,250$ ) and the coupling $\lambda$ (from bottom to top $\lambda=1.0,3.0,5.0)$.

correspond to simulations using the same initial configuration. When $\lambda=1.0$ (bottom row) and for small $T$ a number of small spatially segregated communities can be observed. As $T$ is increased the number of communities increases, but there is no clear spatial organization. The same effect is observed for intermediate values of $\lambda$ (middle row). For sufficiently large $\lambda$ (top row) the system is characterized by the presence of a large cluster, and a number of smaller spatially distributed communities.

When we compare the communities that are formed starting from a random network with the clusters obtained with a local network, a clear effect of disaggregation of the clusters in space can be seen. This is an effect due to the fact that nodes that are connected at random can be located far away from each other. This is expected to have an impact in the local synchronization structure. In order to tackle this issue we analyze the mean frequencies $\Omega$ as a function of the natural frequencies $\omega$ to see if there are synchronized clusters.

In Fig. 8 we plot $\Omega$ vs. $\omega$ for different values of $\lambda$ and $T$. When $\lambda=1.0$ there is only a small dispersion of the natural frequencies. When $\lambda$ is increased these small synchronized clusters coalesce into larger ones. The middle row in Fig. 8 shows that for $\lambda=3.0$ the system is divided into only a few large clusters (middle row). As $\lambda$ is increased further the clusters coalesce into a giant cluster with mean frequency close to zero.

In order to establish a direct relation between the local synchronization and the network structure, in Fig. 9, we plot the nodes in their corresponding spatial location, and use colors to denote their average frequency $\Omega$. As expected, in the bottom row there is almost no synchronization. For $\lambda=3.0$, in the middle row, there is no spatial localization of the synchronized nodes. In some cases there are small local
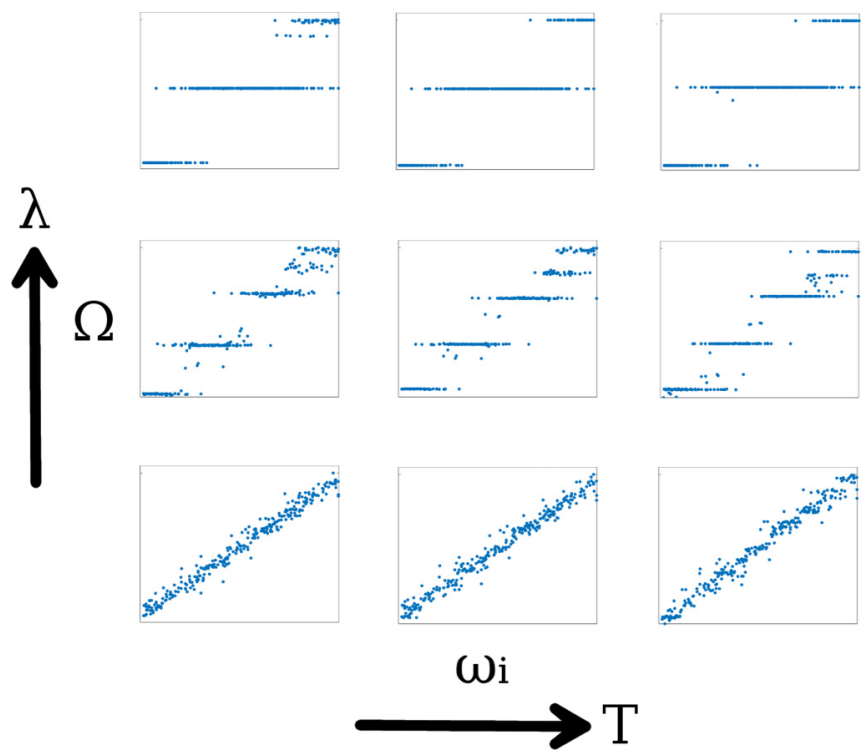

$\omega \mathrm{i}$

FIG. 8. Average frequency $\Omega$ as a function of the initial frequencies $\omega$ for an initial random distribution of connections varying the memory $T$ (from left to right $T=150,180,250$ ) and the coupling $\lambda$ (from bottom to top $\lambda=1.0,3.0,5.0$ ).

clusters, but clearly nodes sharing the same average frequency are dispersed around the whole system. For sufficiently large $\lambda$ a giant cluster emerges, however, as the top row in Fig. 9 shows the smaller clusters are still spatially dispersed.

Finally, we examine the phases of the oscillators, and see how their synchronization is influenced by the underlying network structure. In Fig. 10 we plot a snapshot of the phases in their corresponding spatial localization. In contrast to what was observed for the initial local network (Fig. 5), there are no clusters, and the phases seem to be distributed in space for

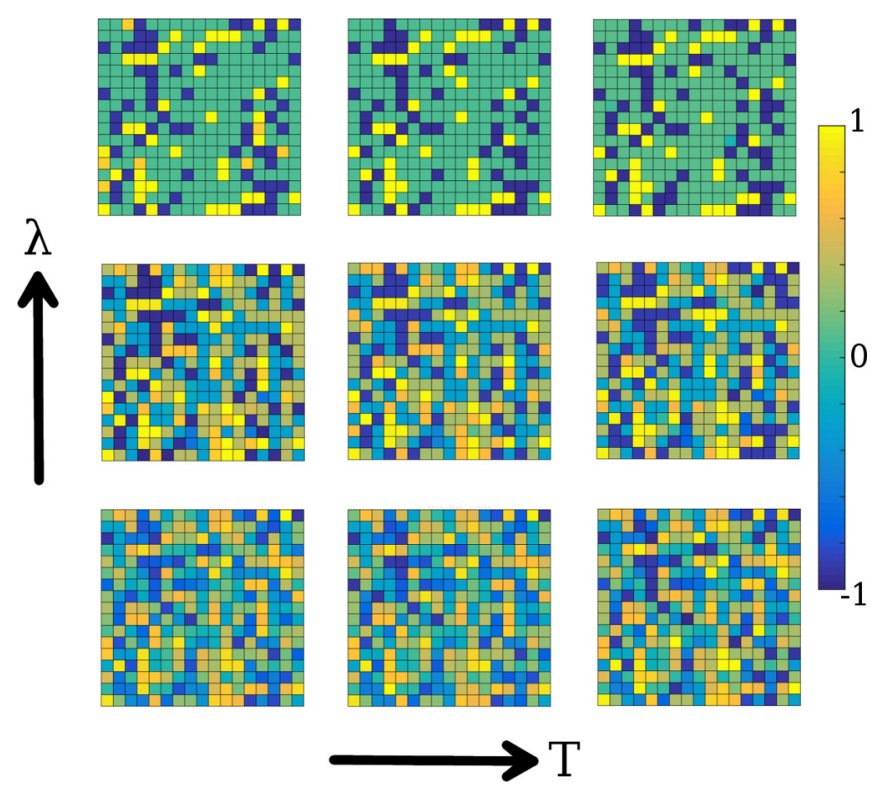

FIG. 9. Average frequency $\Omega$ for an initial random distribution of connections and varying the memory $T$ (from left to right $T=150,180,250$ ) and the coupling $\lambda$ (from bottom to top $\lambda=$ $1.0,3.0,5.0)$. 

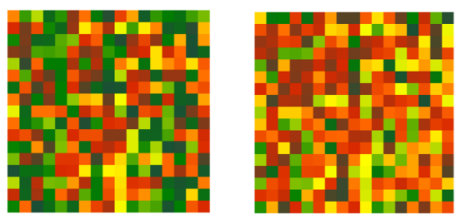

$\lambda$
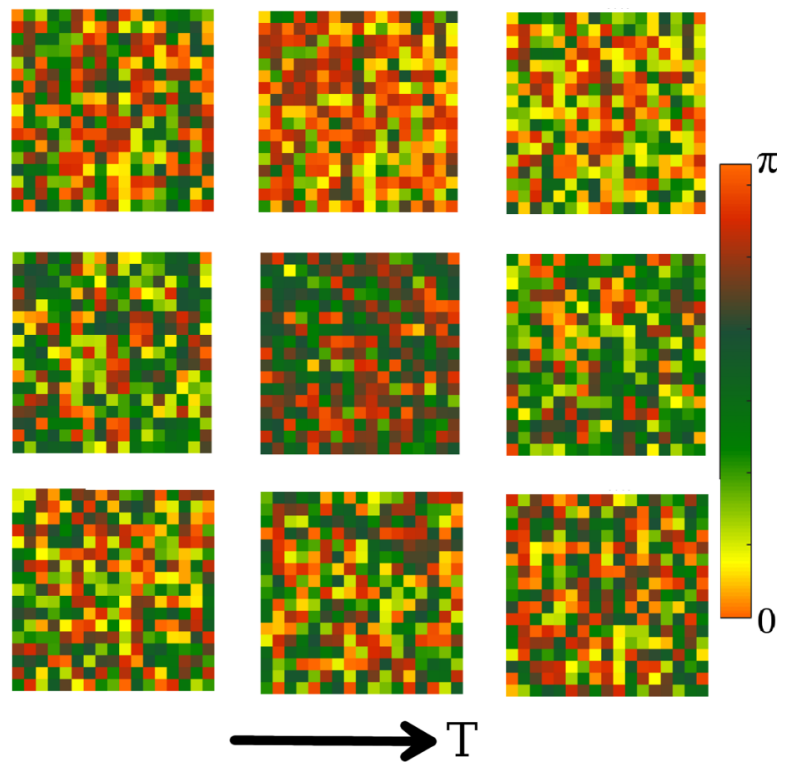

FIG. 10. Phases for an initial random distribution of connections varying the memory $T$ (from left to right $T=150,180,250$ ) and the coupling $\lambda$ (from bottom to top $\lambda=1,3,5$ ).

all the values of $\lambda$. It is interesting to contrast these figures with the behavior of the global order parameter $r$, that evolves to a synchronized state as $\lambda$ is increased (Fig. 6), and also with the frequency synchronized clusters observed in Fig. 9. Clearly there is a synchronization of the system that is not being reflected in the spatial localization of the oscillators.

\section{Scale-free topology}

In the previous sections, we considered local and a random networks, and showed how the underlying topology plays a fundamental role in community formation and also in phase and frequency synchronization. Realistic networks, however, present a complex network structure, characterized by a heterogeneous degree distribution, where a few nodes with a large number of connections play the role of hubs. This is usually approximated by using a scale-free power-law degree distribution. In fact, recently we showed that using a scale-free network and also distance-dependent competing interactions we were able to obtain clustered or striped patterns that quantitatively resemble experimental patterns observed in infant macaques, monkeys, and ferrets [3]. In this section we will take this into account and, as in Ref. [3] we consider a more realistic scale-free topology. and a mexican hat for the interaction function, which has two parameters, the intensity $C$ and the extent of the interaction $\sigma$.

In the Fig. 11 we plot the order parameter $r$ as a function of time $t$ varying the memory $T$ and the mexican hat parameter $C$. All cases shown correspond to an embedded network constructed from a power-law degree distribution with exponent $\alpha=2.1$. The initial conditions for the phases are chosen at random from a uniform distribution in the $[0, \pi)$ interval and the natural frequencies $\omega_{i}$ are randomly chosen from a uniform distribution in the $[-0,5,0,5]$ interval.

In all cases we observe that $r$ reaches a stationary state. For small values of $C$ the order parameter fluctuates around a low
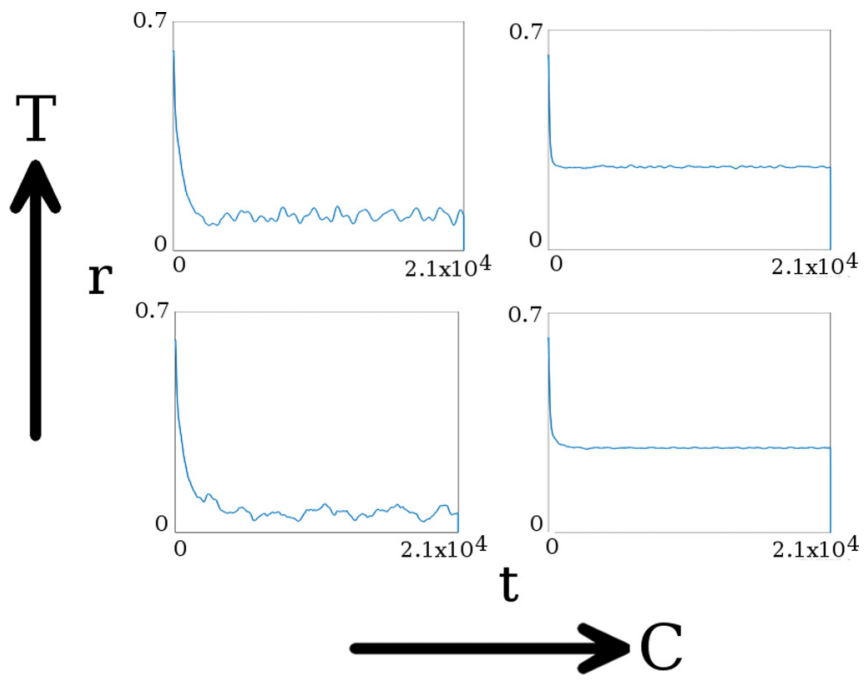

FIG. 11. Order parameter $r$ as a function of time $t$ for an initial scale-free network, varying the memory $T$ (from bottom to top $T=100,300$ ) and the mexican hat parameter $C$ (from left to right $C=1,3)$.

mean value. As $C$ is increased these fluctuation decrease and the stationary value of $r$ increases.

Next, we analyze the community structure. In Fig. 12 we plot the nodes in their corresponding spatial location due to the embedding, where different colors are used to identify the communities. All the figures correspond to simulations using the same initial configuration. In all cases well-defined communities can be observed, resembling the results presented in Fig. 2 for the local topology. Note, however, that in some cases there are a few nodes with different colors inside

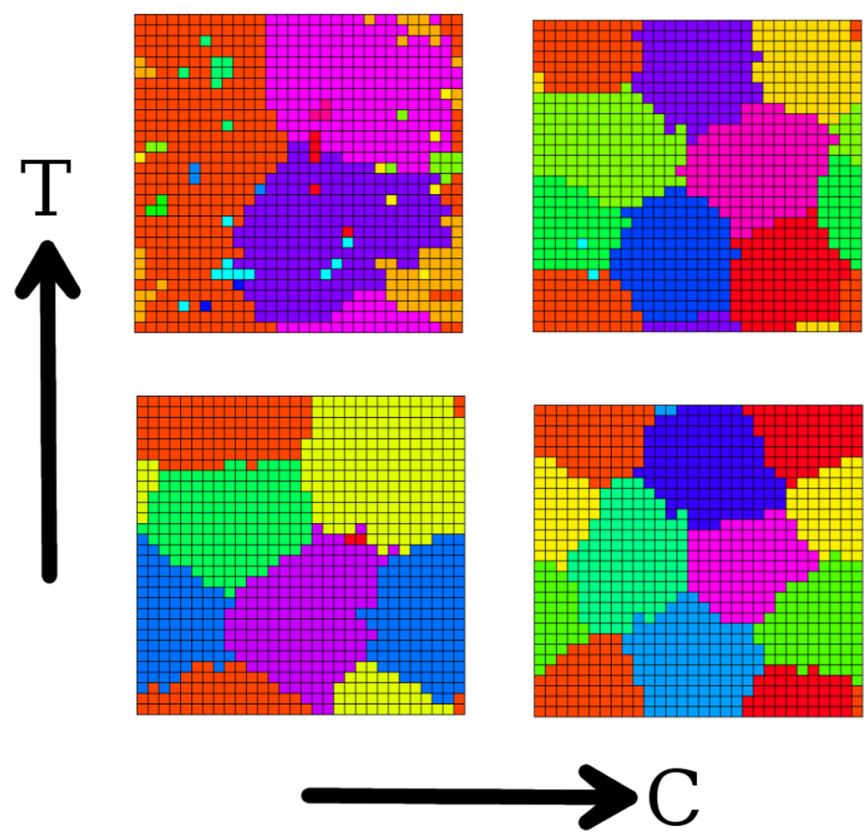

FIG. 12. Communities of interconnected nodes for an initial scale-free network of connections varying the mexican hat parameter $C$ (from left to right $C=1,3$ ) and the memory $T$ (from bottom to top $T=100,300)$. 


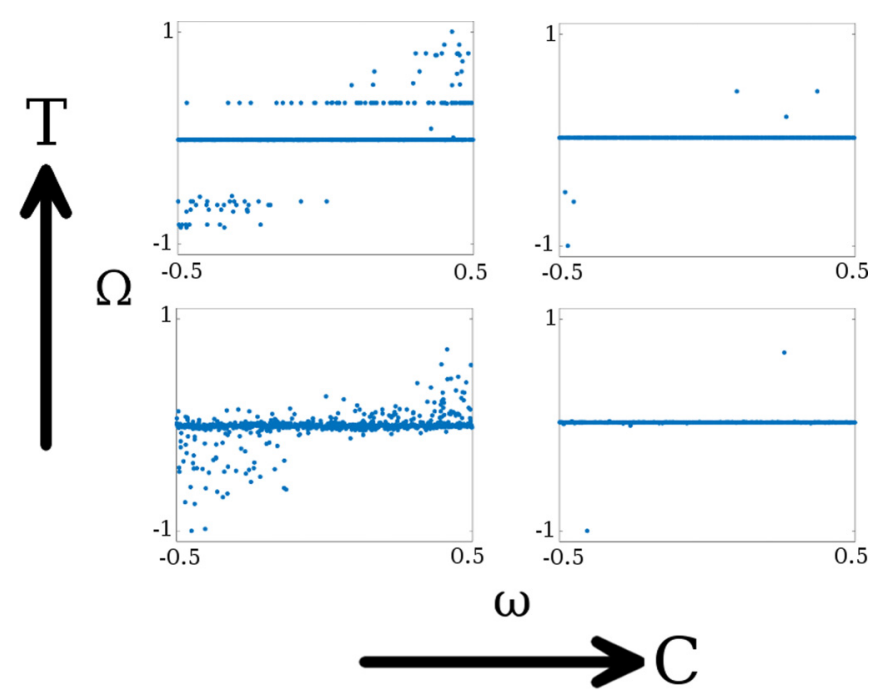

FIG. 13. Average frequency $\Omega$ as a function of the initial frequency $\omega$ for an initial scale-free network of connections, varying the mexican hat parameter $C$ (from left to right $C=1,3$ ) and the memory $T$ (from bottom to top $T=100,300$ ).

the communities. This effect is more clear for $C=1$ and $T=300$, where a mixture of spatially segregated and an interspersed structure is evident. This is an expected effect from embedding a scale-free network, which leads to the presence of many nodes with local connections, and a few nodes with long-range connections between nodes that are far apart.

Next, we plot in Fig. 13 the average frequency $\Omega$ as a function of the initial frequency $\omega$. We find in all cases the presence of a giant synchronized cluster. For $C=1$ there are also nodes with average values outside this cluster, that become synchronized in smaller clusters as $T$ is increased.

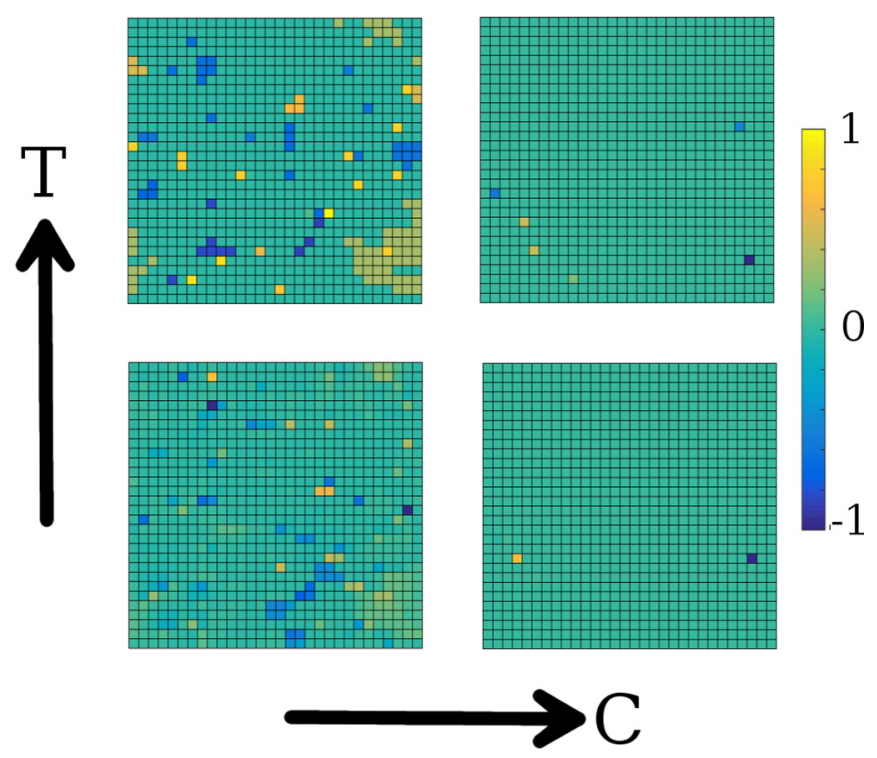

FIG. 14. Spatial location of nodes with average frequency $\Omega$ for an initial scale-free network of connections, varying the mexican hat parameter $C$ (from left to right $C=1,3$ ) and the memory $T$ (from bottom to top $T=100,300)$.

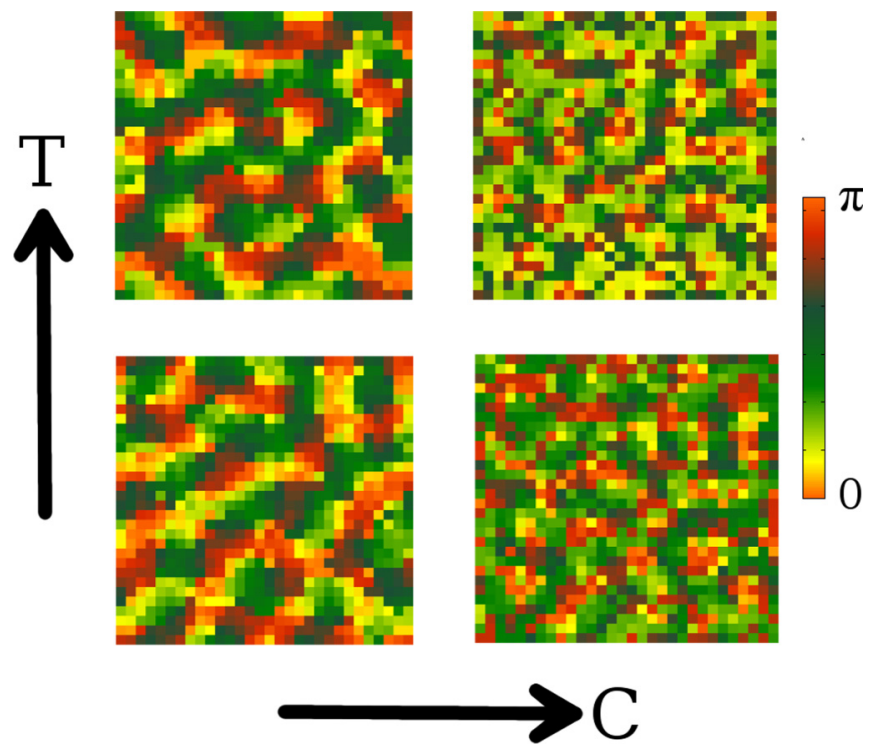

FIG. 15. Snapshot of the phases for an initial scale-free network of connections varying the mexican hat parameter $C$ (from left to right $C=1,3$ ) and the memory $T$ (from bottom to top $T=100,300)$.

In order to establish a direct relation between the local synchronization and the network structure, in Fig. 14, we plot the nodes in their corresponding spatial location, and use colors to denote their average frequency $\Omega$. As expected, all the figures show a spatially homogeneous synchronization, interspersed in some cases by nodes with different average frequencies, in correspondence with the community structure observed in Fig. 12.

Finally, we examine the phases of the oscillators, and see how their synchronization is influenced by the underlying network structure. In Fig. 15 we plot a snapshot of the phases in their corresponding spatial localization. We find that as in Ref. [3] striped patterns emerge.

\section{CONCLUSION}

It is still a matter of study and debate for what reason and in what way salt and pepper maps, observed in some mammals such as mice and squirrels, are an evolutionary strategy that responds to the same environmental problems as the orientation preference maps observed in other mammals such as cats, ferrets, and monkeys. Many animals that manifest the same cortical functional organization inhabit different ecological niches and have different behaviors. For example, galagos are nocturnal, frugivorous, and arboreal primates, while tree shrews are diurnal, insectivorous, and semiarboreal. On the other hand, other animals that share an ecological niche differ in their functional cortical organization, for example the gray squirrel shares many characteristics with the shrew, but does not manifest orientation maps [26]. These differences in functional organization probably are originated in the circuit level of the cortical neurons. Studies in primates that exhibit orientation preference maps (OPM) and rodents that have the salt and pepper organization, reveals that their whole brain networks exhibits different topology arrangements and have different mechanisms for interaction $[33,34]$. 
In this work we presented a simple model based on the spatial patterns observed in the visual cortex. The model incorporates adaptive rules that allow for an interplay between the dynamic of the nodes and an evolving network structure. In this way we can intuitively relate the synchronization properties of phase oscillators, physically located in the spatial positions of the nodes and the underlying network. This can be achieved by embedding the complex network in a two-dimensional Euclidean lattice. The network evolves with rules that modify the weights of the connections. These rules implement plasticity in the weights by means of two conditions: homophily that reinforces dynamically similar states and homeostasis that maintains constant the total weight income from the connections of each node.

We analyze two different underlying network structures: a local and also a random network. In both cases, the system shows the presence of three dynamical regimes: incoherent, periodic, and coherent order. Also, a local analysis of frequency synchronization reveals the presence of synchronized clusters, which can be related to the different regimes. For the incoherent regime there are many small clusters that clearly hinder global synchronization. When the coupling between the oscillators is increased these clusters coalesce, and the system is divided into few clusters with different mean frequencies, whose interaction leads to a period oscillation of the global order parameter. Eventually, as the coupling is increased further these clusters coalesce into a giant clusters, leading to a coherent order.

When we contrast the dynamical behavior of the system with the underlying network we find that spatially located modular structures emerge only for the local network. In the case of the random topology, the rules lead to modules of nodes that are spatially distributed, which reminds the interspersed structures of salt and pepper [26,35].

The results reflect how ordered synchronized states are not necessarily correlated with spatial order. Both for local and random network topologies the system evolves to synchronized states reflected in large values of the global order parameter. Also, a local analysis of the synchronized regimes, using the average frequencies, reveals the presence of synchronized clusters for both topologies. However, this synchronization is not necessarily correlated with a spatially ordered structure. This results suggest that the heterogeneity of the connections plays a fundamental role in the formation of clustered, and also interspersed states, which can emerge from a simple mechanism given by the plasticity of the network.

Finally, we also considered a more realistic system with a scale-free network and competing interactions. Again we find the heterogeneity of the connections plays a fundamental role in the formation of synchronized structures. Even more, we find that the competitive character of the interaction allows for the formation of striped structures as observed in Ref. [3].

\section{ACKNOWLEDGMENTS}

The authors acknowledge support from Universidad Nacional de Córdoba (through SeCyT Grant No. 05/B370), CONICET (under Grant No. PIP 11220150100285) and from CNEA (all Argentinian institutions).
[1] N. Swindale, Cerebral cortex: The singular precision of visual cortex maps, Curr. Biol. 16, 991 (2006).

[2] C. B. Tauro, F. A. Tamarit, and P. M. Gleiser, Synchronization in lattice-embedded scale-free networks, Physica A: Stat. Mech. Appl. 391, 834 (2012).

[3] Y. C. Daza, C. B. Tauro, F. A. Tamarit, and P. M. Gleiser, Modeling spatial patterns in the visual cortex, Phys. Rev. E 90, 042818 (2014).

[4] D. Coppola, L. White, D. Fitzpatrick, and D. Purves, Unequal representation and oblique contours in ferret visual cortex, Proc. Natl. Acad. Sci. USA 95, 2621 (1998).

[5] E. Yacoub, N. Harel, and K. Ugurbil, High-field fmri unveils orientation columns in humans, Proc. Natl. Acad. Sci. USA 105, 10607 (2008).

[6] G. Blasdel and G. Salama, Voltage sensitive dyes reveal a modular organization in monkey striate cortex, Nature (London) 321, 579 (1986).

[7] W. H. Bosking, Y. Zhang, B. Schofield, and D. Fitzpatrick, Orientation selectivity and the arrangement of horizontal connections in tree shrew striate cortex, J. Neurosci. 17, 2112 (1997).

[8] D. H. Hubel and T. N. Wiesel, Receptive fields, binocular interaction and functional architecture of cat striate cortex, J. Physiol. 160, 106 (1962).

[9] D. H. Hubel and T. N. Wiesel, Receptive field and functional architecture of monkey striate cortex, J. Physiol. 125, 215 (1968).
[10] A. Rozenfeld, R. Cohen, D. Ben-Abraham, and S. Havlin, Scale-Free Networks on Lattices, Phys. Rev. Lett. 89, 218701 (2002).

[11] D. Ben-Abraham, A. Rozenfeld, R. Cohen, and S. Havlin, Geographical embedding of scale-free networks, Physica A 330, 107 (2003).

[12] K. D. Miller, Derivation of hebbian equations from a non-linear model, Neural Comp. 2, 319 (1990).

[13] Ch. Von der Malsburg, Self-organization of orientation sensitive cells in the striate cortex, Kybernetik 14, 85 (1973).

[14] N. Swindale, A model for the formation of ocular dominance stripes, Proc. R. Soc. B 208, 243 (1980).

[15] N. Swindale, A model for the formation of orientation columns, Proc. R. Soc. B 215, 211 (1980).

[16] R. Linsker, From basic network principles to neural architecture: emergence of orientation columns, Proc. Natl. Acad. Sci. USA 83, 8779 (1986).

[17] J. S. Espinosa and M. P. Stryker, Development and plasticity of the primary visual cortex, Neuron 75, 230 (2012).

[18] I. J. Gomez Portillo and P. M. Gleiser, An adaptive complex network model for brain functional networks, PloS one 4, e6863 (2009).

[19] P. M. Gleiser and D. H. Zanette, Synchronization and structure in an adaptive oscillator network, Eur. Phys. J. B 53, 233 (2006).

[20] P. M. Gleiser and V. I. Spoormaker, Modelling hierarchical structure in functional brain networks, Philos. Trans. R. Soc. London A 368, 5633 (2010). 
[21] I. J. Gomez Portillo and P. M. Gleiser, A local adaptive algorithm for emerging scale-free hierarchical networks, J. Phys.: Conf. Ser. 246, 012012 (2010).

[22] J. P. Rauschecker and W. Singer, The effects of early visual experience on the cat's visual cortex and their possible explanation by Hebb synapses, J. Physiol. 310, 215 (1981).

[23] S. D. Van Hooser, J. A. Heimel, S. Chung, S. B. Nelson, and L. J. Toth, Orientation selectivity without orientation maps in visual cortex of a highly visual mammal, J. Neurosci. 25, 19 (2005).

[24] K. Ohki, S. Chung, Y. H. Ch'ng, P. Kara, and R. C. Reid, Functional imaging with cellular resolution reveals precise micro-architecture in visual cortex, Nature (London) 433, 597 (2005).

[25] V. Bonin, M. H. Histed, S. Yurgenson, and R. C. Reid, Local diversity and fine-scale organization of receptive fields in mouse visual cortex, J. Neurosci. 31, 18506 (2011).

[26] M. Kaschube, Neural maps versus salt-and-pepper organization in visual cortex, Curr. Opin. Neurobiol. 24, 95 (2014).

[27] J. A. Acebrón, L. L. Bonilla, C. J. Pérez Vicente, F. Ritort, and R. Spigler, The Kuramoto model: A simple paradigm for synchronization phenomena, Rev. Mod. Phys. 77, 137 (2005).
[28] F. A. Rodrigues, T. K. DM. Peron, P. Ji, and J. Kurths, The Kuramoto model in complex networks, Phys. Rep. 610, 1 (2016).

[29] Y. Kuramoto, Chemical Oscillations, Waves and Turbulence, (Springer, Berlin, 1984).

[30] E. Kandel, J. Schwartz, and T. Jensell, Principles of Neural Science (McGraw Hill Medical, New York, 2000).

[31] S. Assenza, R. Gutiérrez, J. Gómez-Gardenes, V. Latora, and S. Boccaletti, Emergence of structural patterns out of synchronization in networks with competitive interactions, Sci. Rep. 1, 99 (2011).

[32] D. B. Vincent, G. Jean-Loup, L. Renaud, and L. Etienne, Fast unfolding of communities in large networks, J. Stat. Mech.: Theory Exp. (2008) P10008.

[33] E. Bullmore and O. Sporns, The economy of brain network organization, Nat. Rev. Neurosci. 13, 336 (2012).

[34] L. Ventura-Antunes, B. Mota, and S. Herculano-Houzel, Different scaling of white matter volume, cortical connectivity, and gyrification across rodent and primate brains, Front. Neuroanat. 7, 3 (2013).

[35] K. Ohki and C. Reid, Specificity and randomness in the visual cortex, Curr. Opin. Neurobiol. 17, 401 (2007). 